\title{
A longitudinal study of child sleep in high and low risk families: relationship to early maternal settling strategies and child psychological functioning
}

Article

Accepted Version

Sheridan, A., Murray, L., Cooper, P. J., Evangeli, M., Byram, V. and Halligan, S. L. (2013) A longitudinal study of child sleep in high and low risk families: relationship to early maternal settling strategies and child psychological functioning. Sleep Medicine, 14 (3). pp. 266-273. ISSN 1389-9457 doi: https://doi.org/10.1016/j.sleep.2012.11.006 Available at https://centaur.reading.ac.uk/36023/

It is advisable to refer to the publisher's version if you intend to cite from the work. See Guidance on citing.

Published version at: http://www.sciencedirect.com/science/article/pii/S1389945712004091\#

To link to this article DOI: http://dx.doi.org/10.1016/j.sleep.2012.11.006

Publisher: Elsevier

All outputs in CentAUR are protected by Intellectual Property Rights law, including copyright law. Copyright and IPR is retained by the creators or other copyright holders. Terms and conditions for use of this material are defined in the End User Agreement. 


\section{www.reading.ac.uk/centaur}

\section{CentAUR}

Central Archive at the University of Reading

Reading's research outputs online 
A longitudinal study of child sleep in high and low risk families: relationship to early maternal settling strategies and child psychological functioning

Dr. Andrew Sheridan

Oxford Radcliffe Hospitals NHS, UK

Prof. Lynne Murray, Prof. Peter J. Cooper

University of Reading, $U K$

Dr. Michael Evangeli, Dr. Victoria Byram

Royal Holloway, University of London

Dr. Sarah Halligan (D.Phil.)

University of Reading, $U K$

Short title: Child sleep development; correlates and consequence

Correspondence:

Sarah L. Halligan

School of Psychology and CLS

University of Reading

3 Earley Gate, Whiteknights

Reading RG6 6AL, UK

Ph.: +44 1183787554

Fax: +44 1183786665

Email: s.1.halligan@ reading.ac.uk 


\begin{abstract}
Objectives: To investigate whether sleep disturbances previously found to characterise high risk infants: a) persist into childhood; b) predict cognitive and emotional/behavioural functioning; and c) are influenced by early maternal settling strategies.

Methods: Mothers experiencing high and low levels of psychosocial adversity (risk) were recruited antenatally and longitudinally assessed with their children. Mothers completed measures of settling strategies and infant sleep postnatally, and at 12- and 18-months infant age. At 5-years, child sleep characteristics were measured via actigraphy and maternal report, and IQ and child adjustment were also assessed.
\end{abstract}

Results: Sleep disturbances observed in high-risk infants persisted at 5-years. Poorer 5-year sleep was associated with concurrent child anxiety-depression and aggression, but there was limited evidence for an influence of early sleep problems. Associations between infant/child sleep characteristics and IQ were also limited. Maternal involvement in infant settling was greater in high risk mothers, and predicted less optimal sleep at 5-years

Conclusions: Early maternal over-involvement in infant settling is associated with less optimal sleep in children, which, in turn, is related to child adjustment. The findings highlight the importance of supporting parents in the early development of good settling practices, particularly in high-risk populations.

Keywords: sleep, longitudinal, child development, parenting, IQ, affective symptoms, aggression 
A longitudinal study of child sleep in high and low risk families: relationship to early maternal settling strategies and child psychological functioning

Previous research has identified associations between family stress or low SES and poor sleep quality in children [1-4]. Collectively, the samples studied have spanned infancy to late childhood, and effect sizes have been small to medium in magnitude. Thus, in a prior report, we examined the development of infant sleep from birth through to 18-months in a longitudinal study of women who were or were not experiencing high levels of psychosocial adversity during pregnancy [1]. Infant sleep characteristics were comparable across high and low adversity groups in the first 3-months of life. However, by 18 months of age, high risk group infants showed more sleep problems (i.e., settling and/or night waking problems, variability in bedtimes and sleep durations) as indexed by both maternal questionnaire and sleep diary. Moreover, psychosocial adversity in our sample was also associated with less optimal maternal settling strategies in early development, which predicted poorer infant sleep at 18 months [1]. The importance of childhood sleep problems is highlighted by existing work which has found that such difficulties are associated with poorer cognitive and behavioural/emotional outcomes [5]. In the cognitive domain, both experimental manipulations of child sleep and observations of naturalistic sleep characteristics have suggested that sleep is an important influence on cognitive functioning. In studies of school aged children, relatively modest experimental reductions (as little as 1-hour) in the amount of sleep have been found to have a detrimental impact on performance on tasks requiring sustained attention, behavioural inhibition, and memory [6-9]. Observational studies have similarly identified a relationship between day-to-day sleep quality and child cognitive functioning [4;5;9]. Notably, there is also some evidence for associations between sleep disturbance and poorer cognitive functioning in the longer term. Thus, reduced academic performance has been observed in the presence of sleep-related breathing disorders [10], as well as in the context of poor sleep duration and/or 
quality in studies of normative samples [5;8]. In addition, a small number of studies have documented concurrent associations between child sleep and trait like cognitive capacities (IQ), although findings have been inconsistent. Specifically, while positive associations between sleep duration and IQ dimensions have been documented in some cases [11;12], others have found longer sleep durations to be associated with lower IQ test scores [13]. The mean age of children in these cross-sectional studies ranged from 6 to 9-years. In addition, a longitudinal study has demonstrated that shorter sleep durations in children aged 8-years predicted lower intellectual ability scores at 10-years (small to medium effect sizes), even taking account of 8year cognitive ability scores [14].

With regard to emotional and behavioural adjustment, sleep difficulties have been associated with the presence of affective (e.g. anxious/depressed) and externalising symptoms in children and adolescents [2;5;15-20]. Although sleep problems may, in part, be symptomatic of underlying disorder [21], there is also evidence to suggest that sleep disturbances themselves contribute to adjustment problems. Medical and behavioural interventions that tackle sleeping difficulties are associated with broader functional improvements [22-25]. Moreover, longitudinal cohort studies have highlighted prospective associations between childhood sleep difficulties and later emotional problems (anxiety and/or depression) and externalising difficulties (attentional problems and aggression) [18;26-30]. These longitudinal effects occur over and above any existing adjustment problems, and samples have included children as young as 4-years and have predicted adjustment as late as adulthood. Nonetheless, some limitations of previous studies have been highlighted, including the use of extremely brief assessments of child sleep quality and/or reliance on a single informant (mothers) to index both sleep and adjustment problems [31;32]. There have also been some notable null findings [28;33]. In particular, in a study incorporating comprehensive assessments of child sleep 
quality, little evidence was found for longitudinal associations with externalising difficulties [34].

A striking feature of research to date is that few studies of younger children and infants have been conducted. A preliminary, small scale study of infants with severe and chronic sleep problems aged 6-12 months found significantly higher rates of ADHD at 5-years relative to a non-sleep disordered comparison group (7/25 versus 0/25) [19]. In addition, one cohort study included brief maternal reports of sleep problems in infancy (6-months) and found limited evidence for an association with attention problems at 5-years and 14-years. Although a stronger pattern of results was obtained for 2-4 year sleep problems, these were measured retrospectively by maternal report at 5-years [35]. A third longitudinal study measured maternal perceptions of infant sleep problems (present/absent on a single item) at 4, 12 and 24-months infant age, and found no prospective associations with child emotional and behavioural adjustment at 6-years [36]. Thus, associations between sleep quality and later adjustment have not consistently been demonstrated when sleep has been studied in infancy and toddlerhood.

The origins of child sleep problems have also been examined. Parental settling strategies have been highlighted as one potentially important influence on sleep development, with more active parental settling behaviours being hypothesised to reduce opportunity for the infant to learn to self-settle [37;38]. Consistent with this view, parental presence or active involvement in settling the child to sleep has been found to be a correlate of poorer sleep quality in the first 3-years of life [37-40]. However, cross-sectional observations are difficult to interpret, since infant sleep difficulties may elicit maternal involvement, as well as the converse [38]. Longitudinal data on this point are extremely limited. In our previous report based on the current sample we found that elevated levels of 'parental presence' settling strategies at 12weeks predicted poor infant sleep at 18-months of age [1] (medium to large effect sizes). Two other longitudinal studies focused on the first 2-years of life have reported similar associations 
[41;42]. However, longer term outcomes associated with early parental settling involvement have not been studied.

In sum, the extant literature highlights associations between sleep problems and adverse cognitive and behavioural-emotional outcomes in children, although studies have mainly relied on parental reports of sleep problems and have focused on school aged children and adolescents. Previous work also links higher levels of parental involvement during the period of settling to sleep with the emergence of sleep problems, but there is limited longitudinal data on this point and studies have focused on the first two years of life. We addressed these issues through an extension of our existing longitudinal study [1], in which we identified early disturbances in sleep characteristics in children growing up in environments characterised by high levels of psychosocial adversity. In the current study, we followed up the same longitudinal sample at 5-years child age and examined whether: a) risk-related disturbances in sleep characteristics that were previously observed at 12 and 18-months of age were also apparent in childhood; b) maternal overinvolved settling strategies early in development predicted persistent sleep disturbances; c) disturbances in child sleep were prospectively and/or concurrently related to child functioning in cognitive, behavioural and emotional domains. With respect to the latter, we were particularly interested in whether observations of associations between sleep disturbances and anxiety/depression, aggression and lower IQ described in the extant literature would be replicated in a younger age group. Given the potential confound that arises between maternal reports of sleep problems and perceptions of child difficulties more generally, we assessed child sleep objectively, using actigraphy, as well as via diary report and questionnaire.

Method 
The study was reviewed by the University of Reading Research and Ethics Committee and the Berkshire NHS Research Ethics Committee (ref 05/Q1602/124). All mothers provided informed consent after a full explanation of study procedures.

Participants

The families who took part in the study belonged to a longitudinal research project described in full in our previous report [1]. Briefly, primiparous mothers attending their routine 20-week antenatal scan appointment at the Royal Berkshire Maternity Hospital in Reading, UK, were screened via a 20 item questionnaire indexing level of psycho-social adversity (questionnaire completion rate $72.5 \%$ ). The questionnaire was specifically developed for the purposes of the current study ${ }^{1}$, based on previous studies of psychosocial adversities in perinatal populations [43-45]. Of 198 women initially contacted based on their questionnaire scores, 67 mothers scoring above a cut off defining the $20 \%$ highest scores (high risk group) were recruited to the study, along with 68 mothers with scores in the $40 \%$ lowest scoring range (low risk group) Cut-off values were pre-established based on pilot work using the screening index. High versus low risk women showed similar recruitment percentages (low risk 60\%, high risk $\left.64 \%: \chi^{2}=0.56, \mathrm{df}=1, \mathrm{~ns}\right)$. Furthermore, we found no significant differences between women from the target sample of 198 who were recruited and those from their group who were not recruited in terms of either original adversity scores $(P \mathrm{~s}>.67)$ or key demographic characteristics (age, marital status, all $P>.26$ ). After excluding those delivering prematurely or withdrawing from the study prior to delivery, numbers in high and low risk groups were 58 (i.e., $87 \%$ of those originally recruited) and $63(93 \%)$ respectively.

On the questionnaire's principal demographic indices of adversity, compared to the low risk group, high risk women were younger [high risk $M=19.7, S D=3.3$, low risk. $M=30.6$, $S D=3.3$ years; $\left.t_{120},=18.3, P<0.001\right]$, more often single [high risk $53.4 \%$, low risk $0 \% ; \chi^{2}$

\footnotetext{
${ }^{1}$ Copy available on request from the corresponding author.
} 
$(1)=45.9, P<0.001]$, and unemployed [high risk $63.2 \%$, low risk $3.1 \% ; \chi^{2}(1)=50.4, P<$ 0.001 ], and fewer were educated beyond 16 years [high risk $14.8 \%$, low risk $96.8 \% ; \chi^{2}(1)=$ $80.8, P<0.001]$. The majority of the sample was white $(86 \%$ of families $)$. Mothers from ethnic minority groups, although relatively few in number, were better represented in the high versus low risk group $\left(21 \%\right.$ vs. $\left.8 \%: \chi^{2}=4.23, \mathrm{df}=1, P=0.04\right)$. Of the original 121 families who were originally recruited to the study, $98(81 \%)$ were retained at 5-years.

\section{Measures}

\section{Child Sleep}

Maternal Reported Problems: At 12-months and 18-months, mothers completed the Infant Sleep Questionnaire (ISQ) [46]. The ISQ is a 10-item parent-report assessment examining infant sleep onset, settling and waking problems, and yields a severity score which has previously proved to be a reliable and valid index of infant sleep problems (in the current study, internal consistency at 12-months $\alpha=.77,18$-months $\alpha=.80$ ). At 5-years, a measure of maternal perceptions of sleep problems was derived from two questions regarding the frequency and severity of child sleep difficulties, each rated from 0 (never/no difficulties) to 3 (every day/severe difficulties). Previous studies have found equivalent, brief, indices to have validity and predictive utility [32;35].

Sleep Diary: At 12-months and 18-months postpartum mothers completed a sleep diary in which they recorded infant wake-time and bed-time for a 7-day period, and the duration of any sleep onset delay. The same measure was also completed at 5-years child age, for a 5-day period during a school week. The Sleep Diary demonstrates good concurrent validity relative to sleep measurement via actigraphy [47]. Based on previous research [1;2], we computed a combined index of disturbance from scores for mean bed-time, bed-time variability, and sleep duration variability. The combined score was computed as the mean of the $z$-scores for each of the individual indicators (standardised scores were used due to large differences in units of 
measurement across variables). This combined index of disturbance, together with the mean hours night-time sleep comprised the primary outcomes from the sleep diaries.

5-year Actigraphy: During the five day period used for the Sleep Diary, children also wore Actiheart physiological monitoring devices (www.camntech.com) around the clock. These acted as an objective measure of the children's nocturnal movements, allowing estimation of whether they were asleep at any given time during the night. The device is worn on the chest, and contains an accelerometer that generates minute-by-minute recordings of movement frequency. The Actiheart has been shown to be a valid and reliable measure of sleep characteristics, such as onset time and duration, when compared to polysomnography [48]. This finding is typical of a wider literature validating Actigraphy as a measure sleep architecture, particularly when complemented by an additional measure such as a sleep diary [49]. Calculations of sleep onset/offset in the current study were based on previously established procedures [4;50]. Key outcome variables were sleep duration and sleep efficiency (i.e., the percentage of sleep period spent sleeping). Actigraphy data were available for 94/98 participants. Missing data resulted from non-completion of the assessment $(n=2)$ or equipment failure/poor data $(n=2)$.

\section{Child Cognitive Ability}

At 5-years, the Wechsler Preschool and Primary Scale of Intelligence - Revised (WPPSIR) [51] were administered in schools. The WPPSI-R has established reliability and validity, is widely used to assess the intelligence of children aged from three years to seven years, and yields indices of performance and verbal IQ, which in combination provide a full IQ score.

\section{$\underline{\text { Child Adjustment }}$}

At 5-years, both mothers and teachers reported on child adjustment on the Child Behavior Checklist (CBCL) (parent and teacher versions) [52]. The CBCL is a widely used questionnaire with established reliability and validity. Due to time constraints, and to maximise comparability 
with previous research [18;28], two subscales were completed: the Anxious/Depressed and Aggression scales. The Anxious/Depressed scale consists of 14 items (e.g., "unhappy, sad, depressed," "cries a lot"), and the Aggression scale consists of 19 items (e.g., "fights," “screams," and "argues"). Combined maternal/teacher report scores were utilised. Previous research has established that combining reports from multiple informants provides a more reliable assessment of child disorder [52;53]. The internal consistencies of the resultant scales were acceptable (Anxious/Depressed $\alpha=.69$, Aggression $\alpha=.89$ ). Scales showed significant skew and were log-transformed to produce appropriate distributions for parametric analyses (for clarity, descriptive statistics for raw scores are reported).

\section{Maternal Settling to Sleep Strategies}

At 4, 7 and 12-weeks, and at 12-months postpartum mothers were asked to complete the self-report Parental Interactive Bedtime Behaviour Scale (PIBBS) [42]. The PIBBS comprises 16 items relating to maternal usage of different strategies to settle their infants to sleep. The principal distinction between items concerns whether or not the parents use a strategy in which they actively involve themselves in settling the infant to sleep, vs. leaving the infant to selfsettle. Three subscales indexing active parental involvement strategies, and derived from factor analysis, were used in our previous report, namely: 'Settle by movement' (e.g., walking in pram, car rides); 'Active comforting' (e.g., stroking, cuddle/rocking, talking softly); and 'Parental presence' (e.g., settle on sofa next to parent, lie next to cot, parental bed) [1]. These same subscales were examined in the current study, with postnatal scores being derived from averaging across 4, 7 and 12-week assessments, and 12-month scores being analysed separately. Each showed good internal consistency postnatally (all $\alpha>.71$ ). At 12-months, internal consistencies for the 'Settle by movement' and 'Parental presence' scales were slightly lower $(\alpha=.69$ and $\alpha=.56$, respectively). However, the scales were retained unchanged to allow comparability with analyses of the postnatal assessments. Finally, since all scales indexed 
parental involvement in the settle to sleep, an overall involvement scale was computed which showed excellent internal consistency at each time point (postnatal $\alpha=.84 ; 12$-months $\alpha=.79$ ).

Analyses

Hypothesis testing was conducted as follows. First, multivariate analysis of variance (MANOVA) was used to examine risk status in relation to sleep diary and actigraphy derived indices of child sleep at 5-years. Maternal perceived sleep difficulties scores from 5-years showed a highly skewed distribution necessitating a separate non-parametric test (MannWhitney U). Second, we used bivariate correlations to examine associations between early maternal settling strategies and child sleep outcomes at 5-years; and follow-up linear regression analyses examined the contribution of maternal settling strategies to variance in child sleep characteristics over and above risk status and/or child gender. Third, correlational analyses were also used to test for prospective (12-month and 18-month sleep) and concurrent associations between sleep characteristics and 5-year IQ/anxious-depression/aggression; and follow-up regression analyses examined whether significant effects were maintained once effects of risk status, and gender where appropriate, were controlled for. Finally, structural equation modeling (SEM) examining pathways from risk status to child adjustment was conducted using Maximum Likelihood Estimation with the MPLUS 6 software (Muthen \& Muthen, 2010). The significance of specific, hypothesized indirect pathways effects was tested within MPLUS using 10,000 bootstrapped resamples to estimate the $95 \%$ confidence interval (CI) for each indirect effect (significant effects are identified where the 95\% CI does not cross zero).

Missing data: As noted, the sample reduced from 121 at recruitment to 98 at 5-year follow-up ( $81 \%$ retention rate). There was more attrition from the high versus the low-risk group (17/58 versus 6/63 at 5-years; $\left.\chi^{2}=7.68, \mathrm{df}=1, P=0.006\right)$. Rates of refusal were similar across groups (3 high versus 2 low risk mothers at 5-years), but a higher proportion of the high 
risk families could not be traced (14 high-risk versus 4 low-risk mothers at 5-years). Therefore, we further examined whether those who dropped out were comparable to those in their group who were retained in terms of initial sample characteristics. ANOVAs comparing scores by retention status while controlling for risk group indicated no significant differences by retention status on any measure; and effect sizes for comparisons on relevant variables were all small (partial $\eta^{2}$ ranged <.001 to .026). Moreover, path analyses were based on the entire recruitment sample (i.e., $n=121$ ) with missing data modelled using full information maximum likelihood estimation, the recommended approach for minimizing the introduction of bias due to missing data $[54 ; 55]$.

\section{Results}

\section{Preliminary analyses}

Descriptive statistics for children in the sample are presented by group in Table 1. High and low risk groups showed similar gender distributions, but there was a trend for the high risk children to be slightly older than their low risk counterparts at 5-years (mean difference 1.4 months, $p=.072$ ), and the high risk group children had lower birth weights [high risk $M=$ $3.30 \mathrm{Kg}, S D=0.57$, low risk $\left.M=3.51, S D=0.50 ; t_{120}=2.09, P<0.05\right]$. These potentially relevant variables were screened as covariates. Age and birth weight were not related to any of the study outcome variables and were not considered further (all $r \mathrm{~s}<.13$ ). Gender effects were also screened for, and significant gender differences were identified in relation to logged CBCL aggression scores (male $M=6.5, S D=3.9$; female $M=3.9, S D=3.3 ; t(96)=2.63, p=.01$ ), sleep period according to diary report (male $M=10.9 \mathrm{hrs,} S D=0.62$; female $M=11.2 \mathrm{hrs}, S D$ $=0.66 ; t(88)=-2.28, p=.025)$ and actigraphy sleep duration (male $M=10.3 \mathrm{hrs}, S D=0.68$; female $M=10.6 \mathrm{hrs}, S D=0.61 ; t(92)=-2.43, p=.017)$. Gender was controlled for in subsequent analyses, as necessary. 
Importantly, consistent with their risk group status, high risk children scored higher on both log transformed aggression and anxiety/depression subscales of the CBCL (combined maternal and teacher report), and they had significantly lower IQ scores than children in the low risk group (Table 1).

Research questions

\section{a) Are risk-related disturbances in sleep characteristics present at 5-years child age?}

Descriptive statistics for sleep outcomes at 5-years are presented in Table 2. MANOVA was used to examine the principal outcomes for the sleep diary and actigraphy (i.e., combined index of disturbance and sleep period according to diary report; sleep duration and efficiency as measured by actigraphy), with risk and child gender as independent variables. Results indicated a significant multivariate effect of risk status $\left(F_{4,86}=5.69, p<.001\right)$, a trend for an effect of gender $\left(F_{4,86}=2.21, p=.075\right)$, and no risk by gender interaction $\left(F_{4,86}=0.36, n s\right)$. Follow-up univariate tests indicated that the effect of risk status was primarily explained by a main effect on the sleep diary disturbance index $\left(F_{1,89}=6.89, p=.010\right)$, where the high risk group showed significantly higher scores (see descriptive statistics in Table 2); other diary/actigraphy dimensions did not attain significance, and there was also no significant group difference in maternal perceptions of child sleep problems $(Z=0.89, P=.37)$.

b) Does maternal over-involvement in infant settling predict child sleep disturbances at 5years?

Correlational analyses examined prospective associations between maternal active involvement settling strategies in the first weeks of life (measured at 4-weeks, 7-weeks and 12weeks postpartum) and at 12-months, and child sleep parameters at 5-years. Results are presented in Table 3. As can be seen from the table, active parental involvement (particularly active soothing and parental presence when infant falling asleep) postnatally tended to be associated with poorer sleep, as evidenced by shorter sleep duration and higher diary 
disturbance index scores. Similar effects emerged for parental involvement at 12-months, although the association with sleep duration was only at trend level of significance. There were no associations with sleep efficiency or maternal perceptions of child sleep problems at 5-years (for maternal perception score with sleep outcomes, all $r_{\mathrm{s}}<.19$, all $p>.076$ ).

Linear regression analyses were used to investigate whether associations between maternal actively involved settling behaviour and child sleep were maintained when controlling for risk status and child gender. First, for the 5-year sleep diary disturbance index, linear regression examined prediction by child gender and risk status in a first step $\left(R^{2}=.092, F_{2,86}=\right.$ $4.36, p=.016)$; only risk status was a significant predictor in the initial model $(B=0.40, S E=$ $0.17, \beta=.25 ; t=2.41, p=.018)$. Maternal actively involved settling (movement, soothing, parental presence at both 1-3 months and 12-months) were entered in a second step, which resulted in a significant improvement in model fit $\left(\Delta R^{2}=.15, \Delta F_{6,80}=2.70, p=.019\right)$. Of the maternal variables, only postnatal active soothing was a significant predictor in the final model $(B=0.014, S E=0.007, \beta=.26 ; t=2.10, p=.038)$. Moreover, the previously significant effect of risk status was reduced to non-significant $(B=0.059, S E=0.22, \beta=.04 ; t=0.27, n s)$, consistent with mediation of the association between risk and sleep outcome by maternal settling behaviour.

Second, equivalent analyses were completed in relation to sleep diary and actigraphy based sleep duration measures. In this case, it was only necessary to control for child gender, as no risk effects were found. With respect to the sleep period according to diary report, linear regression demonstrated that the association with maternal active soothing was maintained $(B=$ $-0.011, S E=0.005, \beta=-.24 ; t=-2.33, p=.022)$ once gender was taken account of $(B=0.33$, $S E=0.13, \beta=-.25 ; t=2.48, p=.015 ;$ model $\left.R^{2}=.11, F_{2,86}=5.54, p=.005\right)$. For actigraphy sleep duration, linear regression examined prediction by child gender in a first step, which was significant $\left(R^{2}=.06, F_{1,91}=6.17, p=.015\right)$. When maternal actively involved settling 
behaviours (postnatal movement, soothing, parental presence; and 12-month movement) were entered in a second step, there was significant improvement in model fit $\left(\Delta R^{2}=.10, \Delta F_{4,87}=\right.$ $2.53, p=.046)$. There were no independently significant predictors in the final model.

c) Are child sleep characteristics prospectively and/or concurrently related to adjustment at 5years?

Correlational analyses tested for prospective associations between 12/18-month sleep indices and child aggression and anxiety at 5-years (as measured by combined mother-teacher report on the CBCL). As indicated in Table 4, there was only a single significant prospective association between infant sleep parameters and log-transformed aggression or anxious/depression scores on the CBCL at 5-years, which was a positive correlation between 12-month ISQ scores and 5-year anxious/depression scores. With respect to concurrent sleep parameters, both anxious/depression and aggression were negatively associated with actigraphy measured sleep duration, and positively associated with maternal perceptions of bedtime difficulties. Log-transformed anxious/depression scores were also positively correlated with sleep diary disturbance scores. There were no other significant effects.

Linear regression examined whether concurrent associations between sleep characteristics (actigraphy sleep duration) and anxious/depression or aggression were maintained once risk status and gender (for aggression) were taken into account. In the prediction of log-transformed CBCL anxious/depression scores, risk status was entered in an initial step $\left(R^{2}=.05, \Delta F_{1,88}=4.13, p=.045\right)$; the addition of 12-month ISQ and 5-year actigraphy sleep duration and diary disturbance index scores explained an additional 10\% of the variance $\left(\Delta R^{2}=.10, \Delta F_{3,85}=3.42, p=.021\right)$. There were trends for independent effects of 5-year sleep duration $(\beta=-.22, t=-1.72, p=.090)$ and 12-month ISQ scores $(\beta=.19, t=1.68$, $p=.098$ ) in the final model. With respect to log-transformed CBCL aggression scores, risk status, gender and actigraphy sleep duration were entered into the regression and explained 
$15 \%$ of the variance $\left(R^{2}=.15, F_{3,90}=5.28, p=.002\right)$. There was a significant independent effect of risk status $(\beta=.25, t=2.50, p=.014)$, as well as trends for effects of gender $(\beta=-.18$, $t=-1.78, p=.079)$ and actigraphy sleep duration $(\beta=-.18, t=-1.76, p=.082)$ in the final model.

d) Are child sleep characteristics prospectively and/or concurrently related to IQ at 5-years?

Prospective and concurrent associations were examined between child sleep parameters and IQ. Results are presented in Table 4. Child IQ scores at 5-years (WPSSI) showed significant inverse associations with 12-month maternal reports of sleep difficulties (ISQ score), and with the combined index of sleep disturbance at 12-months and 18-months. There were no other prospective correlations between sleep parameters and IQ, and nor were there any concurrent associations. Moreover, linear regression indicated that once risk status was controlled for, 12-month and 18-month sleep parameters no longer predicted 5-year IQ $\left(\Delta R^{2}<\right.$ $0.01)$

\section{Analysis of indirect pathways}

The above analyses indicated a link between risk status and maternal active involvement in settling her infant, which in turn was related to shorter actigraphy measured sleep duration at 5-years. Finally, shorter 5-year sleep duration was associated with concurrent child anxiety/depression and aggression scores. Structural equation modelling (SEM) was used to model pathways to child symptoms at 5-years. SEM was conducted using Maximum Likelihood Estimation with the MPLUS 6.0 software (Muthen \& Muthen, 2010). Note that 12month and 18-month sleep parameters did not significantly predict 5-year sleep duration; as they were also not reliably associated with child CBCL scores, they were not included in the model.

The resultant model, depicted in Figure 1, showed a good fit $\left(\chi^{2}=5.20\right.$, df $=4, p=.27$; $\mathrm{CFI}=0.98, \mathrm{TLI}=0.96 ; \mathrm{RMSEA}=0.05,90 \% \mathrm{CI}=0-0.15)$, and all pathways were significant $(p$ 
$<.05$ ). Thus, as well as a direct link between risk status and child adjustment (a latent variable indicated by logged CBCL aggression and anxious/depression scores), an indirect pathway via postnatal maternal settling strategies and child sleep problems was suggested. Analysis of three indirect pathways in the model was completed, with confidence intervals estimated based on 10,000 bootstrapped resamples. First, a partial pathway from risk to 5-year sleep duration via early maternal actively involved settling behavior was found to be significant (indirect effect = $-.146, \mathrm{SE}=.073,95 \% \mathrm{CI}:-.273$ to -.035$)$; a second pathway from postnatal settling behavior to 5-year adjustment via 5-year sleep duration was partially supported (indirect effect $=.003, \mathrm{SE}$ $=.001,95 \%$ CI: .000 to .005$)$; and finally, the complete indirect pathway from risk to 5-year adjustment was also confirmed (indirect effect $=.095, \mathrm{SE}=.060,95 \% \mathrm{CI}: .013$ to .203 ). ${ }^{2}$

\section{Discussion}

The key findings from the current study were as follows. First, previously reported associations between psychosocial adversity and sleep quality in infants in the current sample were maintained in childhood. Second, even once risk group effects were taken into account, maternal settling strategies in the first months of life predicted sleep quality at 5-years. Third, consistent with previous research, there was some evidence of associations between child sleep quality and levels of anxiety/depression and aggression in children; the possibility of links between sleep characteristics and IQ received less support.

In our previous report we observed that although high versus low adversity mothers reported similar levels of sleep problems in their young infants in the first months of life, by 18-months of age significant group differences were apparent [1]. Specifically, high adversity

\footnotetext{
${ }^{2}$ The model depicted in Figure 1 includes a unidirectional pathway from 5-year sleep to child adjustment, as we specifically wished to test potential pathways to child adjustment that operated via child sleep. However, as the association between sleep and adjustment is cross-sectional, this should not be used to infer causality. Indeed, when we tested a second model in which the unidirectional pathway in Figure 1 was replaced with a bidirectional association, this model was also a good fit to the data and the two models were not significantly different to each other $\left(\chi^{2}\right.$ difference test $\left.=2.81, \mathrm{df}=1, \mathrm{~ns}\right)$.
} 
mothers reported more sleep problems on a questionnaire, as well as higher levels of sleep disturbance deriving from diary report (as indexed by later bedtimes and more variable bedtimes and sleep durations). The current paper focused primarily on objective characteristics of sleep at 5-years and, similarly, found more sleep disturbance according to the same diary index in the high vs. the low adversity group; other variables, including sleep duration and efficiency, did not distinguish the groups. Overall, the current findings are consistent with previous reports of links between family stress or low SES and poorer sleep quality in children [2-4]. Importantly, although our analyses utilized groups based on measures of psychosocial adversity taken during the antenatal period, our high adversity group continued to experience multifaceted social difficulties. Thus, at 5-years, compared to their low risk counterparts, highadversity mothers reported experiencing more socioeconomic deprivation, higher levels of affective symptoms, less satisfactory intimate relationships, poorer housing and higher levels of community disorder, and greater levels of adverse life events (data not presented). Associated influences on child sleep characteristics are therefore also likely to be persistent.

Although there are likely to be multiple contributors to an association between sleep parameters and psychosocial adversity, we found maternal settling behaviour to be an important factor. In our previous report, we observed that high risk mothers showed significantly greater involvement in settling their infant to sleep than their low risk counterparts in the post-partum period [1]. Here, we further demonstrated that maternal active involvement in settling the infant in the first 12-weeks of life was a significant predictor of child sleep characteristics at 5-years, and contributed to the association between psychosocial adversity and sleep disturbance at 5-years. Previous longitudinal data on the link between maternal settling strategies and sleep development are extremely limited. Our earlier research with the same sample demonstrated that greater maternal presence at bedtime during the first 3-months predicted poorer sleep at 18-months according to both questionnaire and sleep diary [1]. 
Similarly, Tikotzky and Sadeh [41] found that maternal soothing behaviours at 6-months showed moderate, positive associations with number of night awakenings at 12 months according to maternal report (but not as measured by actigraphy). One further study demonstrated that maternal cognitions likely to enhance parental involvement predicted later child sleep difficulties and maternal settling behaviour [56].

Our findings represent a significant advance on existing studies, indicating that associations between early maternal settling behaviour and infant sleep persist into childhood. Active maternal involvement during the settle to sleep in the postnatal weeks was a key predictor of child sleep at 5-years, being associated with greater overall sleep disturbance and shorter sleep duration as indexed by both diary report and actigraphy. Importantly, early infant sleep characteristics did not appear to be able to explain this effect, and therefore our data were consistent with a causal influence of maternal behaviour on infant sleep development. The negative association observed between active parental settling behaviour and infant sleep is assumed to arise because of reduced opportunity for the infant to learn to self-settle [38]. Our observations suggest that early high levels of parental involvement have persistent effects, presumably due to the establishment of poor sleep patterns and/or ongoing suboptimal parental behaviour [41].

In our previous report, we found that high adversity mothers were less likely to adjust their settling strategies with infant maturation in the post-partum period [1]. Such observations may be a consequence of limited social support and advice from others; and also limited physical resources, particularly lack of space, which may make reducing involvement in settling the infant particularly challenging. In addition, our high risk mothers reported elevated symptoms of anxiety and depression, which may have impinged on their ability to respond in a sensitive and flexible way towards their infant [57-59]. Supporting parents in the development of good practices for facilitating infant self-settling in the first year of life may be particularly 
important for high risk groups, as our research suggests that persistent child sleep disruption is more likely to arise in this context.

We also examined links between early and concurrent poor sleep quality, and child emotional and cognitive functioning at 5-years of age. Previous research has indicated longitudinal associations between sleep problems and later affective and externalising difficulties in children. However, the majority of previous studies have relied on self or maternal reports of sleep difficulties and adjustment problems, and have studied child aged 4years and older [31;32]. We found only limited evidence for longitudinal associations between sleep problems at 12 or 18-months and child adjustment at 5-years, with just a single significant association being found between maternal reported difficulties (ISQ score) at 12-months and 5year anxiety/depression. However, concurrent associations were demonstrated between poorer sleep and anxiety-depression and aggression at 5-years, including an inverse association between actigraphy measured sleep duration and 5-year difficulties.

The lack of a strong pattern of associations between sleep characteristics in the second year of life and later child adjustment is in contrast to previous reports of longitudinal associations between poor sleep and adjustment problems in older children [31]. However, a recent study spanning a similar age range to our own (4-months to 6-years) also failed to find longitudinal associations between infant sleep and later child emotional and behavioural difficulties [36]. Sleep undergoes significant development during the first 2-years of life, and problems are relatively common during this period. As such, early difficulties may primarily reflect transient individual differences in normative development. However, by 5-years sleep difficulties are less common and may be indicative of more stable problems. Notably, we found tentative evidence for an indirect pathway from early maternal active involvement in settling the infant to sleep to poorer child adjustment, via shorter 5-year sleep duration. Previous research using a genetically informative design has indicated that associations between sleep 
difficulties and child adjustment may largely be explained by shared environmental characteristics [60]. The current findings highlight family level adversities and specific maternal behaviours as relevant psychosocial factors.

We also examined associations between early/concurrent sleep quality and child cognitive ability (5-year IQ). Although preliminary analyses indicated that sleep disturbance as measured by sleep diary at 12 and 18-months was associated with lower 5-year IQ, correlations were not maintained once risk group status was taken into account. There were no concurrent associations between sleep quality and IQ. Thus, while our observations are broadly in support of a small number of existing studies which have indicated that poorer sleep is associated with lower IQ [11;12], including longitudinally [14], our findings highlight the need to take full account of socio-demographic characteristics which may be associated with both variables.

Our study has some notable strengths, including the longitudinal design, the inclusion of early developmental observations, and the objective measurement of sleep quality at 5-years. The use of actigraphy was important, as a stronger set of findings emerged when actual sleep duration versus diary reported sleep period was the dependent variable, presumably due to more precise measurement via actigraphy. However, there were also some limitations. First, some attrition occurred in our sample, particularly in the high risk group. Although we did not find evidence to suggest that high risk families who were lost to follow up differed from their counterparts who were retained, we cannot rule out the possibility that they differed in ways that were not measured. Second, the inclusion of direct measurements of sleep characteristics meant that our sample size was necessarily of modest size relative to some previous studies in this area, and we consequently did not have sufficient power to adjust our analyses to correct for multiple comparisons or to detect small effects. Third, our analyses focused on maternal involvement during the settle to sleep. Although previous research has identified this as being a key correlate of infant sleep, an examination of strategies that actively promote infant self- 
settling (such as leaving to cry for brief periods) is also desirable. Finally, although longitudinal analyses may suggest causal relationships, ours is still, essentially, a correlational design; intervention studies are required to confirm causal effects. Interpretation of the associations between sleep and adjustment is particularly problematic, as here only cross-sectional effects were found. Nonetheless, our data are consistent with a potential role for early maternal settling strategies in influencing the development of child sleep, and thereby contributing to adjustment. Future research should investigate whether the promotion of effective maternal settling strategies in the first years of life is associated with better child outcomes, particularly focusing on high risk populations where persistent child sleep disturbances appear more likely to occur. If proven effective, such interventions could help ameliorate the potentially deleterious effects of poor sleep in later development. 


\section{Reference List}

[1] Cronin A, Halligan SL, Murray L. Maternal psychosocial adversity and the longitudinal development of infant sleep. Infancy 2008;13(5):469-495.

[2] Bates JE, Viken RJ, Alexander DB, Beyers J, Stockton L. Sleep and adjustment in preschool children: Sleep diary reports by mothers relate to behavior reports by teachers. Child Dev 2002;73(1):62-74.

[3] Buckhalt JA, El-Sheikh M, Keller P. Children's sleep and cognitive functioning: race and socioeconomic status as moderators of effects. Child Dev 2007;78(1):213-231.

[4] Sadeh A, Raviv A, Gruber R. Sleep patterns and sleep disruptions in school-age children. Dev Psychol 2000;36(3):291-301.

[5] Jenni OG, Dahl RE. Sleep, cognition and emotion: a developmental view. In: Nelson CA, Luciana M, editors. Handbook of developmental cognitive neuroscience. 2 ed. Cambridge, MA: MIT Press, 2008: 801-817.

[6] Sadeh A, Gruber R, Raviv A. The effects of sleep restriction and extension on schoolage children: what a difference an hour makes. Child Dev 2003;74(2):444-455.

[7] Fallone G, Acebo C, Arnedt JT, Seifer R, Carskadon MA. Effects of acute sleep restriction on behavior, sustained attention, and response inhibition in children. Percept Mot Skills 2001;93(1):213-229.

[8] Curcio G, Ferrara M, De GL. Sleep loss, learning capacity and academic performance. Sleep Med Rev 2006;10(5):323-337.

[9] Kopasz M, Loessl B, Hornyak M, Riemann D, Nissen C, Piosczyk H, et al. Sleep and memory in healthy children and adolescents - a critical review. Sleep Med Rev 2010;14(3):167-177.

[10] Blunden S, Lushington K, Kennedy D. Cognitive and behavioural performance in children with sleep-related obstructive breathing disorders. Sleep Med Rev 2001;5(6):447-461.

[11] Paavonen EJ, Raikkonen K, Pesonen AK, Lahti J, Komsi N, Heinonen K, et al. Sleep quality and cognitive performance in 8-year-old children. Sleep Med 2010;11(4):386392.

[12] Touchette E, Petit D, Seguin JR, Boivin M, Tremblay RE, Montplaisir JY. Associations between sleep duration patterns and behavioral/cognitive functioning at school entry. Sleep 2007;30(9):1213-1219.

[13] Geiger A, Achermann P, Jenni OG. Association between sleep duration and intelligence scores in healthy children. Dev Psychol 2010;46(4):949-954.

[14] Buckhalt JA, El-Sheikh M, Keller PS, Kelly RJ. Concurrent and longitudinal relations between children's sleep and cognitive functioning: the moderating role of parent education. Child Dev 2009;80(3):875-892. 
[15] Kheirandish L, Gozal D. Neurocognitive dysfunction in children with sleep disorders. Developmental Science 2006;9(4):388-399.

[16] Fallone G, Owens JA, Deane J. Sleepiness in children and adolescents: clinical implications. Sleep Medicine Reviews 2002;6(4):287-306.

[17] Chervin RD, Dillon JE, Archbold KH, Ruzicka DL. Conduct problems and symptoms of sleep disorders in children. J Am Acad Child Adolesc Psychiatry 2003;42(2):201208.

[18] Gregory AM, O'Connor TG. Sleep problems in childhood: a longitudinal study of developmental change and association with behavioral problems. J Am Acad Child Adolesc Psychiatry 2002;41(8):964-971.

[19] Thunstrom M. Severe sleep problems in infancy associated with subsequent development of attention-deficit/hyperactivity disorder at 5.5 years of age. Acta Paediatr 2002;91(5):584-592.

[20] Wiggs L, Montgomery P, Stores G. Actigraphic and parent reports of sleep patterns and sleep disorders in children with subtypes of attention-deficit hyperactivity disorder. Sleep 2005;28(11):1437-1445.

[21] Owens JA. The ADHD and sleep conundrum: A review. J Dev Behav Pediatr 2005;26(4):312-322.

[22] Galland BC, Dawes PJ, Tripp EG, Taylor BJ. Changes in behavior and attentional capacity after adenotonsillectomy. Pediatr Res 2006;59(5):711-716.

[23] Minde K, Faucon A, Falkner S. Sleep problems in toddlers: effects of treatment on their daytime behavior. J Am Acad Child Adolesc Psychiatry 1994;33(8):1114-1121.

[24] Chervin RD, Ruzicka DL, Giordani BJ, Weatherly RA, Dillon JE, Hodges EK, et al. Sleep-disordered breathing, behavior, and cognition in children before and after adenotonsillectomy. Pediatrics 2006;117(4):e769-e778.

[25] Sadeh A. Cognitive-behavioral treatment for childhood sleep disorders. Clinical Psychology Review 2005;25(5):612-628.

[26] Wong MM, Brower KJ, Zucker RA. Childhood sleep problems, early onset of substance use and behavioral problems in adolescence. Sleep Med 2009;10(7):787-796.

[27] Quach J, Hiscock H, Canterford L, Wake M. Outcomes of child sleep problems over the school-transition period: Australian population longitudinal study. Pediatrics 2009;123(5):1287-1292.

[28] Gregory AM, Caspi A, Eley TC, Moffitt TE, Oconnor TG, Poulton R. Prospective longitudinal associations between persistent sleep problems in childhood and anxiety and depression disorders in adulthood. J Abnorm Child Psychol 2005;33(2):157-163.

[29] Gregory AM, Van der Ende J, Willis TA, Verhulst FC. Parent-reported sleep problems during development and self-reported anxiety/depression, attention problems, and aggressive behavior later in life. Arch Pediatr Adolesc Med 2008;162(4):330-335. 
[30] Gregory AM, Rijsdijk FV, Lau JY, Dahl RE, Eley TC. The direction of longitudinal associations between sleep problems and depression symptoms: a study of twins aged 8 and 10 years. Sleep 2009;32(2):189-199.

[31] Gregory AM, Sadeh A. Sleep, emotional and behavioral difficulties in children and adolescents. Sleep Med Rev 2011.

[32] Gregory AM, Cousins JC, Forbes EE, Trubnick L, Ryan ND, Axelson DA, et al. Sleep items in the child behavior checklist: a comparison with sleep diaries, actigraphy, and polysomnography. J Am Acad Child Adolesc Psychiatry 2011;50(5):499-507.

[33] Johnson EO, Chilcoat HD, Breslau N. Trouble sleeping and anxiety/depression in childhood. Psychiatry Res 2000;94(2):93-102.

[34] El-Sheikh M, Kelly RJ, Buckhalt JA, Benjamin HJ. Children's sleep and adjustment over time: the role of socioeconomic context. Child Dev 2010;81(3):870-883.

[35] O'Callaghan FV, Al MA, O'Callaghan M, Clavarino A, Williams GM, Bor W, et al. The link between sleep problems in infancy and early childhood and attention problems at 5 and 14 years: Evidence from a birth cohort study. Early Hum Dev 2010;86(7):419-424.

[36] Price AM, Wake M, Ukoumunne OC, Hiscock H. Outcomes at six years of age for children with infant sleep problems: longitudinal community-based study. Sleep Med 2012;13(8):991-998.

[37] Adair R, Bauchner H, Philipp B, Levenson S, Zuckerman B. Night waking during infancy: role of parental presence at bedtime. Pediatrics 1991;87(4):500-504.

[38] Sadeh A, Tikotzky L, Scher A. Parenting and infant sleep. Sleep Med Rev 2010;14(2):89-96.

[39] Anders TF, Halpern LF, Hua J. Sleeping through the night: a developmental perspective. Pediatrics 1992;90(4):554-560.

[40] Sadeh A, Mindell JA, Luedtke K, Wiegand B. Sleep and sleep ecology in the first 3 years: a web-based study. J Sleep Res 2009;18(1):60-73.

[41] Tikotzky L, Sadeh A. Maternal sleep-related cognitions and infant sleep: a longitudinal study from pregnancy through the 1st year. Child Dev 2009;80(3):860-874.

[42] Morrell J, Cortina-Borja M. The developmental change in strategies parents employ to settle young children to sleep, and their relationship to infant sleeping problems, as assessed by a new questionnaire: The Parental Interactive Bedtime Behaviour Scale. Infant and Child Development 2002;11(1):17-41.

[43] Conroy S, Marks MN. Maternal psychological vulnerability and early infant care in a sample of materially disadvantaged women. Journal of Reproductive and Infant Psychology 2003;21(1):5-22.

[44] Cooper PJ, Murray L, Hooper R, West A. The development and validation of a predictive index for postpartum depression. Psychol Med 1996;26(3):627-634. 
[45] Brooke H, Gibson A, Tappin D, Brown H. Case-control study of sudden infant death syndrome in Scotland, 1992-5. BMJ 1997;314(7093):1516-1520.

[46] Morrell JM. The Infant Sleep Questionnaire: A new tool to assess infant sleep problems for clinical and research purposes. Clinical Psychology and Psychotherapy 1999;4(1):20-26.

[47] Rogers AE, Caruso CC, Aldrich MS. Reliability of sleep diaries for assessment of sleep/wake patterns. Nurs Res 1993;42(6):368-372.

[48] Gögenur I, Petersen M, Kücükakin B, Rosenberg J, Wildschiøtz G. Evaluation of a combined actigraphy and heart rate variability monitor for determining sleep and wake in healthy adults. Somnologie 2006;10(3):143-155.

[49] Sadeh A. The role and validity of actigraphy in sleep medicine: an update. Sleep Med Rev 2011;15(4):259-267.

[50] Lotjonen J, Korhonen I, Hirvonen K, Eskelinen S, Myllymaki M, Partinen M. Automatic sleep-wake and nap analysis with a new wrist worn online activity monitoring device vivago WristCare. Sleep 2003;26(1):86-90.

[51] Wechsler D. Wechsler Preschool and Primary Scale of Intelligence - Revised. San Antonio, TX: The Psychological Corporation, 1989.

[52] Achenbach TM, Rescorla LA. Manual for the ASEBA School-Age Forms and Profiles. Burlington, VT: University of Vermont, Research Center for Children, Youth and Families, 2001.

[53] Jensen PS, Salzberg AD, Richters JE, Watanabe HK. Scales, diagnoses, and child psychopathology: I. CBCL and DISC relationships. J Am Acad Child Adolesc Psychiatry 1993;32(2):397-406.

[54] Schafer JL, Graham JW. Missing data: our view of the state of the art. Psychol Methods 2002;7(2):147-177.

[55] Allison PD. Missing data techniques for structural equation modeling. J Abnorm Psychol 2003;112(4):545-557.

[56] Tikotzky L, Shaashua L. Infant sleep and early parental sleep-related cognitions predict sleep in pre-school children. Sleep Med 2012;13(2):185-192.

[57] Field T. Early interactions between infants and their postpartum depressed mothers. Infant Behav Dev 1984;7:537-540.

[58] Murray L, FioriCowley A, Hooper R, Cooper P. The impact of postnatal depression and associated adversity on early mother-infant interactions and later infant outcome. Child Dev 1996;67(5):2512-2526.

[59] Halligan SL, Cooper PJ, Fearon RMP, Sampson S, Crosby M, Murray L. The longitudinal development of emotion regulation capacities in children at risk for externalizing disorders. Dev Psychopathol. In press 2012. 
[60] Gregory AM, Eley TC, O'Connor TG, Plomin R. Etiologies of associations between childhood sleep and behavioral problems in a large twin sample. J Am Acad Child Adolesc Psychiatry 2004;43(6):744-751. 